Arthur A. Sagle*

Nagoya Math. J.

Vol. 39 (1970), 81-106

\title{
SOME HOMOGENEOUS EINSTEIN MANIFOLDS
}

\author{
ARTHUR A. SAGLE*
}

1. Introduction. Let $G$ be a connected Lie group and $H$ a closed subgroup with Lie algebra $\mathfrak{h}$ such that in the Lie algebra $g$ of $G$ there exists a subspace $\mathfrak{m}$ with $\mathfrak{g}=\mathfrak{m}+\mathfrak{h}$ (subspace direct sum) and $[\mathfrak{m} \mathfrak{h}] \subset \mathfrak{m}$. In this case the corresponding manifold $M=G / H$ is called a reductive homogeneous space and $(\mathfrak{g}, \mathfrak{h})$ (or $(G, H))$ a reductive pair. In this paper we shall show how to construct invariant pseudo-Riemannian connections on suitable reductive homogeneous spaces $M$ which make $M$ into an Einstein manifold. Thus from the formula for the connection at the point $H \in M$ we compute the Ricci tensor, $\operatorname{Ric}(X, Y)$ for $X, Y \in \mathfrak{m}$, and show $-4 \operatorname{Ric}(X, Y)=\eta C(X, Y)$ where $C(X, Y)$ is the pseudo-Riemannian metric inducing the connection and $\eta$ is a suitable real number.

In the second section we shall review the algebra necessary to compute various formulas concerning $\operatorname{Ric}(X, Y)$. Also we shall note that up to a scalar multiple the pseudo-Riemannian connections are in one-to-one correspondence with elements of a certain symmetric space $\mathfrak{N}$ which is a subset of a Jordan algebra, and a connection of the first kind is obtained from the identity of the Jordan algebra (which is in $\mathfrak{R}$ ).

In the third section we consider reductive pairs $(\mathfrak{g}, \mathfrak{h})$ with $\mathfrak{g}$ simple and $\mathfrak{h}$ semi-simple and develop a new decomposition of the subspace $\mathfrak{m}$ into ad h-invariant subspace by a process of "taking centralizers". Using this decomposition we construct pseudo-Riemannian connections on $M=G / H$ which are holonomy irreducible provided the connection of the first kind is holonomy irreducible on $M$ (which is the case). Thus we have found a solution to the following type of deformation problem in the symmetric space $\mathfrak{R}$ : Let $M=G / H$ be a suitable reductive homogeneous space such that the connection of the first kind is a holonomy irreducible pseudo-Riemannian connection given by the identity $I \in \mathfrak{R}$. Then how can $I$ be deformed

Received October 18, 1968.

* Supported in part by NSF Grant GP-6364. 
in a continuous manner to an element $S \in \mathfrak{N}$ such that the corresponding pseudo-Riemannian connection is irreducible? We also give a construction of the corresponding simply connected reductive homogeneous space with this connection.

Next in section three we develop the general equations for the above reductive homogeneous space to be Einstein. Then in section four we give general examples e.g. $S O(n) / S O(k)$ with $k<n-1$ which are irreducible Einstein manifolds. Also we give a general test to see if the connection is of the first kind-usually it is not. These and other examples lead to the following conjecture: Let $G / H$ be a simply connected reductive homogeneous space with $G$ simple and $H$ semi-simple. Then there exists an invariant pseudo-Riemannian connection on $G / H$ which makes it into a holonomy irreducible Einstein manifold.

\section{Algebraic preliminaries.}

Let $M=G / H$ be a reductive homogeneous space with the corresponding reductive pair $(\mathfrak{g}, \mathfrak{h})$. In the decomposition $\mathfrak{g}=\mathfrak{m} \dot{+} \mathfrak{h}$ the subspace $\mathfrak{m}$ is related to invariant connections on $G / H$ by the following result of Nomizu [5].

Theorem. Let $G / H$ be a reductive homogeneous space with a fixed Lie algebra decomposition $\mathfrak{g}=\mathfrak{m} \dot{+} \mathfrak{h}$ such that $[\mathfrak{h} \mathfrak{m}] \subset \mathfrak{m}$. Then there exists a one-to-one correspondence between the set of all $G$-invariant connections $\nabla$ on $G / H$ and the set of all bilinear functions $\alpha: \mathfrak{m} \times \mathfrak{m} \rightarrow \mathfrak{m}$ such that for $X, Y \in \mathfrak{m}($ Ad $h) \alpha(X, Y)=\alpha(($ Ad $h) X$, $($ Ad $h) Y$ ) for all $h \in H$.

Thus if we let $(\mathfrak{m}, \alpha)$ denote the algebra with vector space $\mathfrak{m}$ and multiplication $\alpha(X, Y)$ for $X, Y \in \mathfrak{m}$, then for every $h \in \mathfrak{h}$ the mappings $D(h): \mathfrak{m} \rightarrow \mathfrak{m}: Z \rightarrow\left[\begin{array}{ll}h & Z\end{array}\right]$ are derivations of the algebra (m, $\left.\alpha\right)$. Using the notation in $[3,8]$ let $a(X): \mathfrak{m} \rightarrow \mathfrak{m}: Y \rightarrow \alpha(X, Y)$ and $b(X): \mathfrak{m} \rightarrow \mathfrak{m}: Y \rightarrow \alpha(Y, X)$ and $L(X): \mathfrak{m} \rightarrow \mathfrak{m}: Y \rightarrow X Y$ where $X Y=[X Y]_{\mathfrak{m}}$ is the projection of $[X Y]$ in $\mathrm{g}$ into $\mathfrak{m}$. Then from [5] we have the curvature and torsion formulas for the corresponding connection $\nabla$ given by

$$
\begin{aligned}
& R(X, Y)=[a(X), a(Y)]-a(X Y)-D(h(X, Y)) \\
& \operatorname{Tor}(X, Y)=[a(X)-b(X)-L(X)] Y
\end{aligned}
$$

where $h(X, Y)=[X, Y]_{\mathfrak{h}}$ is the projection of $[X, Y]$ in $g$ into $\mathfrak{h}$.

Let $(\mathfrak{m}, X Y)$ denote the algebra with vector space $\mathfrak{m}$ and multiplication 


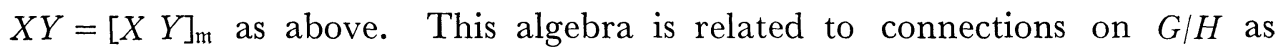
follows [5].

THEOREM. Let $G / H$ be a reductive homogeneous space with corresponding decomposition $\mathfrak{g}=\mathfrak{m} \dot{+} \mathfrak{h}$, then there exists one and only one $G$-invariant connection which has zero torsion and such that a one-parameter subgroup $x(t)$ generated by $X \in \mathfrak{m}$ projects by $\pi: G \rightarrow G / H: x(t) \rightarrow x^{*}(t)$ into a geodesic $x^{*}(t)$ in $G / H$. In this case $\alpha(X, Y)=1 / 2 X Y$ and the connection is called the canonical connection of the first kind on $G / H$.

In $[3,8]$ general pseudo-Riemannian connections given by the algebras $(\mathfrak{m}, \alpha)$ are compared with pseudo-Riemannian connections of the first kind. Thus let $C(X, Y)$ be a nondegenerate symmetric bilinear form on $\mathfrak{m}$ which satisfies

$$
\begin{aligned}
& C(D(h) X, Y)=-C(X, D(h) Y) \text { and } \\
& C(\alpha(Z, X), Y)=-C(X, \alpha(Z, Y))
\end{aligned}
$$

for all $X, Y, Z \in \mathfrak{m}$ and $h \in \mathfrak{h}$. Then the algebra ( $\mathfrak{m}, \alpha)$ induces pseudoRiemannian connection on $G / H$ and from [4] we have

$$
\alpha(X, Y)=\frac{1}{2} X Y+U(X, Y)
$$

where $U(X, Y)$ is a commutative multiplication on $m$. In the case of pseudo-Riemannian connections we summarize the results on holonomy $[3,7,8,9]$.

TheORem 1. Let $G / H$ be a simply connected reductive homogeneous space with fixed decomposition $\mathfrak{g}=\mathfrak{m}+\mathfrak{h}$. Let $B(X, Y)$ (resp. $C(X, Y)$ ) be a nondegenerate form which induces an invariant pseudo-Riemannian connection of the first kind (resp. arbitrary kind) and let $(\mathfrak{m}, X Y)($ resp. $(\mathfrak{m}, \alpha))$ denote the corresponding algebras. Then

(1) the holonomy algebra (= Lie algebra of the holonomy group) of the connection determined by $C(X, Y)$ is generated by all the maps $a(X)$ and $D(h)$. Denote this holonomy algebra by hol $(C)$.

(2) If $G / H$ is not a symmetric space and is holonomy irreducible, then $(\mathfrak{m}, \alpha)$ is a simple algebra and hol $(C)$ is contained in the Lie algebra generated by all the maps $a(X)$ and $b(Y)$ for all $X, Y \in \mathfrak{m}$. Furthermore $(\mathfrak{m}, \alpha)$ has no left ideals which are $D(\mathfrak{h})$-invariant (i.e. $\mathfrak{m}$ is hol $(C)$-irreducible).

(3) Suppose $G / H$ is not a symmetric space and suppose the algebra ( $\mathfrak{m}, X Y$ ) 
together with the form $B(X, Y)$ determine a pseudo-Riemannian connection of the first kind. If $G / H$ is holonomy irreducible relative to this connection, then (m, $X Y$ ) is a simple algebra. The converse holds if $G / H$ is simply connected. In this case hol $(B)$ equals the Lie algebra generated by all the maps $L(X)$ for $X \in \mathfrak{m}$.

(4) If $G / H$ is not a symmetric space and the corresponding reductive pair $(\mathfrak{g}, \mathfrak{h})$ is such that $\mathfrak{g}$ is simple and $\mathfrak{h}$ is semi-simple, then $\mathfrak{g}=\mathfrak{m}+\mathfrak{h}$ where $\mathfrak{m}=\mathfrak{h} \perp$ relative to the Killing form of $\mathfrak{g}$. Furthermore $(\mathfrak{m}, X Y)$ is a simple algebra which has nondegenerate invariant form $B(X, Y)$ equal to the Killing form of $\mathrm{g}$ restricted to $\mathfrak{m} \times \mathfrak{m}$.

Thus Theorem 1(4) indicates it is natural to start with a pseudoRiemannian connection of the first kind on $G / H$ and try to compare other pseudo-Riemannian connections to this one. Some results of this nature are obtained in $[3,8]$ and we need the following. From [4] the algebra (m, $X Y)$ and the form $C(X, Y)$ determine the algebra $(\mathfrak{m}, \alpha)$ by

$$
\begin{aligned}
2 C(Z, \alpha(X, Y)) & =C(Z, X Y)+C(Z X, Y)+C(X, Z Y) \\
& =-2 C(\alpha(X, Z), Y) .
\end{aligned}
$$

Next suppose $B(X, Y)$ induces a connection of the first kind and $C(X, Y)$ induces another connection, then since these forms are nondegenerate there exists $S \in G L(\mathfrak{m})$ such that

$$
C(X, Y)=B(S X, Y) \text {. }
$$

Since $C$ and $B$ are $D(\mathfrak{h})$-invariant symmetric forms $S$ also satisfies

$$
[S, D(h)]=0 \text { all } h \in \mathfrak{h} \text { and }
$$

$$
S^{b}=S\left(=S^{c}\right) \text {. }
$$

where $b$ and $c$ denote adjoints relative to $B$ and $C$. Conversely if $S=S^{b} \in G L(\mathfrak{m})$ satisfies (2.7), then we can define a pseudo-Riemannian metric and connection by the formulas (2.5) and (2.6). Note that from (2.5) and (2.6) we obtain an exact formula for $(\mathfrak{m}, \alpha)$ in terms of $(\mathfrak{m}, X Y)$ and $S[3,8]$ :

$$
\begin{aligned}
& 2 \alpha(X, Y)=X Y+S^{-1}[X(S Y)-(S X) Y] \text { or } \\
& 2 a(X)=L(X)+S^{-1}[L(X) S-L(S X)] .
\end{aligned}
$$

Also note that the set of $S$ 's in Hom ( $\mathfrak{m}, \mathfrak{m})$ satisfying (2.7) and (2.8) form a Jordan algebra $(\mathfrak{A}, \cdot)$ relative to the usual multiplication $2 S_{1} \cdot S_{2}=S_{1} S_{2}+S_{2} S_{1}$. 
Thus the set $\mathfrak{N}=\mathfrak{U} \cap G L(\mathfrak{m})$ which yield connections is a manifold which is actually a symmetric space [4] and the connection of the first kind is given by $S=\lambda I \in \mathfrak{A}$.

We now compute the various formulas needed. For $X, Y, Z \in \mathfrak{m}$ let

$$
\sigma(X, Y) Z=D(Z, Y) X
$$

where $D(U, V)=D(h(U, V))$, then from $[6,9]$ we have for

$B(X, Y)=$ Killing form $\mid \mathfrak{m} \times \mathfrak{m} \equiv \operatorname{Kill}(X, Y)$ that

Kill $(X, Y)=\operatorname{tr} L(X) L(Y)+2 \operatorname{tr} \sigma(X, Y)$.

Also from $[6,7]$ other identities for the algebra $(\mathfrak{m}, X Y)$ are

$$
X Y=-Y X \quad \text { (bilinear) }
$$

$$
D(X, Y)=-D(Y, X) \text { (bilinear) }
$$

$$
D(X, Y) Z+D(Y, Z) X+D(Z, X) Y=X(Y Z)+Y(Z X)+Z(X Y)
$$

$$
D(X Y, Z)+D(Y Z, X)+D(Z X, Y)=0
$$

$$
[D(h), D(X, Y)]=D(D(h) X, Y)+D(X, D(h) Y)
$$

$$
D(h)(X Y)=(D(h) X) Y+X(D(h) Y) .
$$

We use some of these to compute $\operatorname{Ric}(X, Y)$. Thus if we set

$$
\Sigma(U, V) W=R(W, V) U
$$

we have from (2. 1), that

$$
\Sigma(U, V)=b(\alpha(V, U))-a(V) b(U)+b(U) L(V)-\sigma(U, V) .
$$

In particular when $\operatorname{Tor}(X, Y)=0$ we have from (2.2) that $b(X)=a(X)-L(X)$ and from (2. 4) we have $a(X)^{c}=-a(X)$ and $L(X)^{b}=-L(X)$. Thus $\operatorname{tr} a(X)=$ $\operatorname{tr} L(X)=0$ so that $\operatorname{tr} b(X)=0$. Consequently the Ricci tensor is given by

$$
\begin{aligned}
\operatorname{Ric}(U, V) & =\operatorname{tr} \sum(U, V) \\
& =\operatorname{tr} b(\alpha(V, U))-\operatorname{tr} b(U) a(V)+\operatorname{tr} b(U) L(V)-\operatorname{tr} \sigma(U, V) \\
& =-\operatorname{tr} b(U)[a(V)-L(V)]-\operatorname{tr} \sigma(U, V) \\
& =-\operatorname{tr} b(U) b(V)-\operatorname{tr} \sigma(U, V) .
\end{aligned}
$$

Next, using $b(X)=a(X)-L(X)$ and (2.10) we recompute Ric $(U, V)$ in terms of $S$ as follows. First we note 


$$
2 b(X)=-L(X)+S^{-1}[L(X) S-L(S X)]
$$

so that

$$
\begin{aligned}
\text { (2. 20) }-4 \operatorname{Ric}(U, V)= & \operatorname{tr}\left(-L(U)+S^{-1}[L(U) S-L(X U)]\right) \cdot\left(-L(V)+S^{-1}[L(V) S-L(S V)]\right) \\
& +4 \operatorname{tr} \sigma(U, V) \\
=2 & \operatorname{tr} L(U) L(V)+\operatorname{tr} S^{-1} L(S U) S^{-1} L(S V) \\
& \quad-\operatorname{tr} L(U) S^{-1} L(V) S-\operatorname{tr} L(V) S^{-1} L(U) S+4 \operatorname{tr} \sigma(U, V) \\
=2 & \operatorname{Kill}(U, V)-2 \operatorname{tr} L(U) S^{-1} L(V) S \\
& +\operatorname{tr} S^{-1} L(S U) S^{-1} L(S V)
\end{aligned}
$$

using (2. 12) and

$$
\begin{aligned}
\operatorname{tr} L(V) S^{-1} L(U) S & =\operatorname{tr}\left[L(V) S^{-1} L(U) S\right]^{b} \\
& =\operatorname{tr} S^{b} L(U)^{b}\left(S^{-1}\right)^{b} L(V)^{b} \\
& =\operatorname{tr} S L(U) S^{-1} L(V) \\
& =\operatorname{tr} L(U) S^{-1} L(V) S .
\end{aligned}
$$

\section{Equations for an Einstein manifold.}

If we want $G / H$ to be an Einstein manifold relative to the connection induced by some nondegenerate form $C(X, Y)$, we want a real number $\eta$ so that $-4 \operatorname{Ric}(X, Y)=\eta C(X, Y)$. So we consider the form symmetric and bilinear in $X, Y \in \mathfrak{m}$ given by

$$
f(X, Y, S, \eta)=\eta C(X, Y)+4 \operatorname{Ric}(X, Y)
$$

and attempt to solve the following equation for $S$ and $\eta$ for all $X, Y \in \mathfrak{m}$ :

$$
\begin{aligned}
0 & =f(X, Y, S, \eta) \\
& =\eta B(S X, Y)+4 \operatorname{Ric}(X, Y) .
\end{aligned}
$$

In particular if $B(X, Y)=\mathrm{Kill}(X, Y)$ is an admissible nondegenerate form on $\mathfrak{m}$, we must solve the equation

$$
\begin{gathered}
f(X, Y, S, \eta)=\operatorname{Kill}((\eta S-2 I) X, Y)+2 \operatorname{tr} L(X) S^{-1} L(Y) S \\
-\operatorname{tr} L(S X) S^{-1} L(S Y) S^{-1}=0
\end{gathered}
$$

In theory these equations can be expressed by equations in the Jordan algebra $\mathfrak{A}$ as follows. We note that the three symmetric bilinear forms 


$$
\begin{aligned}
& g_{1}(X, Y)=\operatorname{Kill}(X, Y), g_{2}(X, Y)=\operatorname{tr} L(X) S^{-1} L(Y S) \\
& \text { and } g_{3}(X, Y)=\operatorname{tr} L(S X) S^{-1} L(S Y) S^{-1}
\end{aligned}
$$

are related to $B(X, Y)$ by

$$
g_{i}(X, Y)=B\left(T_{i} X, Y\right)
$$

where $T_{i}=T_{i}(S) \in \operatorname{Hom}(\mathfrak{m}, \mathfrak{m})$. But since

$$
[S, D[h)]=0 \text { and } L(D(h) X)=[D(h), L(X)]
$$

(using (2. 18)), we obtain

$$
g_{i}(D(h) X, Y)=-g_{i}(X, D(h) Y) .
$$

This yields $\left[T_{i}, D(h)\right]=0$ as follows:

$$
\begin{aligned}
B\left(D T_{i} X, Y\right) & =-B\left(T_{i} X, D Y\right) \\
& =-g_{i}(X, D Y) \\
& =g_{i}(D X, Y) \\
& =B\left(T_{i} D X, Y\right) .
\end{aligned}
$$

Also $T_{i}^{b}=T_{i}$ so that $T_{i} \in \mathfrak{A}$. Thus equation (3.2) is equivalent to the following equation in $\mathfrak{A}$ :

$$
\eta S-2 T_{1}(S)+2 T_{2}(S)-T_{3}(S)=0
$$

Unfortunately the functions $T_{i}$ are difficult to find so we now proceed using equation (3.3) directly. We are starting with a nonsymmetric pair $(\mathfrak{g}, \mathfrak{h})$ with $\mathfrak{g}$ simple and $\mathfrak{h}$ semi-simple. This yields that the representation $a d_{g} \mathfrak{h}$ in $\mathfrak{g}$ is faithful so that by $[2$, p. 69-70] the trace form Kill $\mid \mathfrak{h} \times \mathfrak{h}$ is nondegenerate on $\mathfrak{h}$. Thus we may decompose

$$
\mathfrak{g}=\mathfrak{m}+\mathfrak{h} \text { (subspace direct sum) }
$$

where $\mathfrak{m}=\mathfrak{h} \perp$ relative to the Killing form. From this we see $[\mathfrak{h} \mathfrak{m}] \subset \mathfrak{m}$ so relative to this decomposition $(\mathfrak{g}, \mathfrak{h})$ is a reductive pair and $\mathrm{Kill} \mid \mathfrak{m} \times \mathfrak{m}$ is a nondegenerate form with $\operatorname{Kill}(X Y, Z)=\operatorname{Kill}(X, Y Z)$ and $\operatorname{Kill}(D(h) X, Y)=$ - Kill $(X, D(h) Y)$. Now let $K=\{X \in \mathfrak{m}: D(h) X=0$ for all $h \in \mathfrak{h}\}$, then using (2. 18) and (2.15) we see that $K$ is a Lie subalgebra of $(\mathfrak{m}, X Y)$. Next we note

$$
h(K, \mathfrak{m})=0,
$$


for if $W \in K, Z \in \mathfrak{m}$ we have $\left[\begin{array}{ll}W & Z\end{array}\right]=W Z+h(W, Z)$ and for $h \in \mathfrak{h}$

$$
\begin{aligned}
0 & =\operatorname{Kill}_{g}\left(\left[\begin{array}{ll}
h & W
\end{array}\right], Z\right) \\
& =\operatorname{Kill}_{g}\left(h,\left[\begin{array}{ll}
W & Z
\end{array}\right]\right) \\
& =\operatorname{Kill}_{g}(h, h(W, Z)), \text { using } \mathfrak{m}=\mathfrak{h} \perp .
\end{aligned}
$$

Thus since Kill $\mid \mathfrak{h} \times \mathfrak{h}$ is nondegenerate, $h(W, Z)=0$. This yields $[K \mathfrak{m}]=$ $K \mathfrak{m} \subset \mathfrak{m}$ so that $\mathfrak{m}$ is $K$-module relative to $a d_{g} K$ and also relative to multiplication in $(\mathfrak{m}, X Y)$. To see this let $V, W \in K, Z \in \mathfrak{m}$ then from (3. 4) and (2. 15) we have

$$
(V W) Z+(W Z) V+(Z V) W=0
$$

so that $L(K)=\{L(V): V \in K\}$ is a Lie algebra of linear transformations on m. Thus $\mathfrak{m}$ is an $L(K)$-module and $a d_{\mathfrak{m}} V=L(V)$ for $V \in K$.

Next we recall some facts on centralizers [2, p. 102]. Let $T$ be a subset of a Lie algebra $L$ of linear transformations on a finite dimensional vector space $V$ over the reals. Then the centralizer $C_{L}(T)=\{W \in L:[W, h]=0$ for all $h \in T\}$. We have the following result.

Theorem. Let $L$ be a completely reducible Lie algebra of linear transformations in the finite dimensional vector space $V$ and let $L_{1}$ be a completely reducible subalgebra of $L$, then the centralizer $C_{L}\left(L_{1}\right)$ is completely reducible subalgebra of $L$ acting on $V$.

For our applications, let $V=\mathfrak{g}, L=a d \mathfrak{g}$ and $L_{1}=a d \mathfrak{h}$. Then since $g$ and $\mathfrak{h}$ are semi-simple, $L$ and $L_{1}$ are completely reducible on $g$ and therefore $C_{L}\left(L_{1}\right)$ is completely reducible on g. But

$$
\begin{aligned}
C_{L}\left(L_{1}\right) & =\{a d W \in a d \mathfrak{g}:[a d \mathfrak{h}, \text { ad } W]=0\} \\
& =\{a d W \in a d g: a d[\mathfrak{h} W]=0\} .
\end{aligned}
$$

But since $g$ is simple, and $a d[\mathfrak{h} W]=0$ yields $[\mathfrak{g} W]=0$. Next let $W=W_{1}+W_{2}$ where $W_{1} \in \mathfrak{m}, W_{2} \in \mathfrak{h}$, then $0=\left[\mathfrak{h} W_{1}\right]+\left[\mathfrak{h} W_{2}\right]$ which implies by the direct sum $\mathfrak{g}=\mathfrak{m}+\mathfrak{h}$ that $\left[\mathfrak{h} W_{i}\right]=0$. But since $\mathfrak{h}$ is semi-simple $\left[\mathfrak{h} W_{2}\right]=0$ implies $W_{2}=0 ;$ thus $W=W_{1} \in \mathfrak{m}$. This gives

$$
C_{L}\left(L_{1}\right)=a d_{g} K
$$

is completely reducible in $g$ and consequently completely reducible on $a d_{\mathfrak{g}} K$-submodules. Thus, writing $\mathfrak{g}=\mathfrak{h}+\mathfrak{m}$ we have for $W \in K$ the matrix 


$$
a d_{\mathrm{g}} W=\left[\begin{array}{cc}
0 & 0 \\
0 & W_{22}
\end{array}\right]
$$

using $[K \mathfrak{m}] \subset \mathfrak{m}$. From this we see that the action of $a d_{\mathfrak{g}} K$ and $L(K)$ on $\mathfrak{m}$ are the same (given by $W_{22}$ ). Thus since $a d_{\mathfrak{g}} K$ is completely reducible on $\mathfrak{m}$, so is $L(K)$. Thus from [2, p. 47] we may write $L(K)=L(c) \oplus L\left(K^{\prime}\right)$ where the center $L(c)=\{L(A) \in L(K):[L(A), L(K)]=0\}$ and $L\left(K^{\prime}\right)=\{L(V) \in$ $\left.L(K): V \in K^{\prime}\right\}$ is a semi-simple ideal or zero. Thus since $K \rightarrow L(K)$ is an isomorphism (because $L(K) \cong a d_{g}(K)$ and $a d_{g} X=0$ implies $X=0$ ) we may conclude

$$
K=c \oplus K^{\prime}
$$

where $K^{\prime}$ is zero or semi-simple and $c$ is the center of $K$.

Next we show that Kill $\mid K \times K$ is nondegenerate. Since $h$ is semi-simple and $\mathfrak{h} \rightarrow D(\mathfrak{h}): h \rightarrow D(h)$ is a homomorphism (actually an isomorphism using (2. 17) and the simplicity of $\mathfrak{g}$, we have $D(\mathfrak{h})$ is semi-simple. Thus $D(\mathfrak{h})$ is completely reducible on $\mathfrak{m}$ and since $K$ is $D(\mathfrak{h})$-invariant we may write $\mathfrak{m}=K \dot{+} b$ where $b$ is $D(\mathfrak{h})$-invariant complement. Now $D(\mathfrak{h}) b=b$ as follows: if $p=D(\mathfrak{G}) b$ is properly contained in $b$ then $p$ is a porper $D(\mathfrak{G})$-invariant submodule of $b$ (because $p \subset b$ yields $D(\mathfrak{h}) p \subset D(\mathfrak{h}) b=p$ ). But $D(\mathfrak{h})$ is completely reducible on $b$ therefore there exists a $D(h)$-invariant complement $p^{\prime}$ with $b=p+p^{\prime}$. But $D(\mathfrak{h}) p^{\prime} \subset p^{\prime} \cap D(\mathfrak{G}) b=p^{\prime} \cap p=0$; thus we must have $p^{\prime} \subset K$. This contradiction shows $D(\mathfrak{h}) b=b$ and $\mathfrak{m}=K+D(\mathfrak{h}) b$.

Using this result we assume there is $U \in K$ with $\operatorname{Kill}(U, K)=0$ then

$$
\begin{aligned}
\operatorname{Kill}(U, \mathfrak{m}) & =\operatorname{Kill}(U, D(\mathfrak{h}) b) \\
& =\operatorname{Kill}(D(\mathfrak{h}) U, b)=0 .
\end{aligned}
$$

Thus since Kill $\mid \mathfrak{m} \times \mathfrak{m}$ is nondegenerate we have $U=0$. In particular Kill $\mid c \times c$ and Kill $\mid K^{\prime} \times K^{\prime}$ are nondegenerate forms and $\operatorname{Kill}\left(c, K^{\prime}\right)=0$. For this we just note

$$
\begin{aligned}
\operatorname{Kill}\left(c, K^{\prime}\right) & =\operatorname{Kill}\left(c, K^{\prime} K^{\prime}\right) \\
& =\operatorname{Kill}\left(c K^{\prime}, K^{\prime}\right)=0 .
\end{aligned}
$$

We can now decompose $\mathfrak{m}=K \dot{+} b$ where we can now assume $b=K^{\perp}$ relative to the Killing form and $b$ is $D(\mathfrak{h})$-invariant (using $\operatorname{Kill}(D X, Y)=$ $-\operatorname{Kill}(X, D Y))$ and Kill $\mid b \times b$ is nondegenerate. Next let 


$$
K_{1}=\{Y \in b: L(U) Y=0 \text { for all } U \in K\} .
$$

Then noting from (2. 15) that for $W \in K$ and $X, Y \in \mathfrak{m}$,

$$
W(X Y)=(W X) Y+X(W Y)
$$

we see that $L(K)$ is a Lie subalgebra of the derivation algebra of ( $m, X Y$ ). In particular this gives $K_{1}$ is a subalgebra of ( $\mathfrak{m}, X Y$ ) which is $L(K)$ and $D(\mathfrak{h})$-invariant (using (2.18)).

We shall now show Kill $\mid K_{1} \times K_{1}$ is nondegenerate. Since $\mathfrak{m}$ is $L(K)$ completely reducible the $L(K)$-submodule $b$ is $L(K)$-completely reducible. Thus we decompose $b=K_{1}+K_{2}$ into $L(K)$-submodules. Now in a manner similar to the proof of $b=D(\mathfrak{h}) b$ we can show $K_{2}=L(K) K_{2}$. Consequently if we assume for $P \in K_{1}$ that $\operatorname{Kill}\left(P, K_{1}\right)=0$ then

$$
\operatorname{Kill}\left(P, K_{2}\right)=\operatorname{Kill}\left(P, L(K) K_{2}\right)=\operatorname{Kill}\left(L(K) P, K_{2}\right)=0 .
$$

Thus $\operatorname{Kill}(P, b)=0$ so that $P=0$ since Kill $\mid b \times b$ is nondegenerate.

Thus we can decompose $b=K_{1} \dot{+} K_{2}$ where $K_{2}=K_{1} \perp$ relative to the Killing form and $K_{2}$ is $L(K)$ and $D(\mathfrak{h})$-invariant and Kill $\mid K_{2} \times K_{2}$ is nondegenerate. Due to the lack of more identities, which lead to Lie modules, this process of taking centralizers appears to stop at this point; we summarize some of the results.

Proposition 2. (a) Let $\mathrm{g}$ be a simple Lie algebra and h a semi-simple Lie subalgebra, then $\mathfrak{g}$ can be decomposed $\mathfrak{g}=\mathfrak{m} \dot{+} \mathfrak{g}$ where $\mathfrak{m}=\mathfrak{h} \perp$ relative to the Killing form and $(\mathfrak{g}, \mathfrak{h})$ is a reductive pair.

(b) If $(\mathfrak{g}, \mathfrak{h})$ is not a symmetric pair then the algebra $(\mathfrak{m}, X Y)$ is simple [9] and $\mathfrak{m}$ has the orthogonal decomposition (relative to the Killing form) $\mathfrak{m}=c+K^{\prime}+K_{1} \dot{+} K_{2}$ where the subspaces are all $D(\mathfrak{G})$-invariant and satisfy the multiplicative relations

$$
\begin{aligned}
& c c=c K^{\prime}=c K_{1}=0, \quad c K_{2} \subset K_{2} \\
& K^{\prime} K^{\prime}=K^{\prime}, \quad K^{\prime} K_{1}=0, \quad K^{\prime} K_{2} \subset K_{2} \\
& K_{1} K_{1} \subset K_{1}, \quad K_{1} K_{2} \subset K_{2}, \quad K_{2} K_{2} \subset \mathfrak{m}
\end{aligned}
$$

(c) Furthermore $K=c \oplus K^{\prime}$ is a Lie subalgebra of $(\mathfrak{m}, X Y)$ such that $D(\mathfrak{h}) K=0$ and $K^{\prime}$ is semi-simple or zero.

(d) $\mathfrak{h}+K$ is a Lie subalgebra of $\mathfrak{g}$ such that $\mathfrak{n}$ is an $a d(\mathfrak{h}+K)$-module and $a d(\mathfrak{h}+K)$ are derivations of $(\mathfrak{m}, X Y)$. Also $D(\mathfrak{h}) K_{i}=K_{i}$ for $i=1,2$ and $L(K) K_{2}=K_{z}$ and $K_{2}$ is ad(h) $\left.+K+K_{1}\right)$-invariant. 
We have shown all but the last part of (d) and this follows from $h\left(K_{1}, K_{2}\right)=0$. To see this note

$$
\begin{aligned}
& 0=\operatorname{Kill}\left(\left[\mathfrak{L} K_{1}\right], K_{2}\right) \text {, using } K_{2}=K_{1} \perp \\
& =\operatorname{Kill}\left(\mathfrak{h},\left[\begin{array}{ll}
K_{1} & K_{2}
\end{array}\right]\right) \\
& =\operatorname{Kill}\left(\mathfrak{h}, h\left(K_{1}, K_{2}\right)\right) \text {, using } \mathfrak{n t}=\mathfrak{h} \perp \text {. }
\end{aligned}
$$

We now use this decomposition to make a guess for a matrix of the linear transformation $S$ which determines a connection via an algebra ( $\mathfrak{m}, \mathfrak{a})$. If in practice some of the above spaces are zero, then the corresponding elements of the matrix are just omitted. When $K^{\prime}$ is semi-simple, let $K^{\prime}=K_{1}^{\prime} \oplus \cdots \oplus K_{t}^{\prime}$ and let $S$ have matrix given by:

$$
\begin{aligned}
& S\left|c=s_{1} I, \quad S\right| K_{j}{ }^{\prime}=s_{2 j} I \\
& S\left|K_{1}=s_{3} I, \quad S\right| K_{2}=s_{4} I
\end{aligned}
$$

where $I$ denotes the identity matrix of appropriate size and $s_{i}, s_{2 j} \neq 0$.

Now recall that if $K^{\prime}=K_{1}^{\prime} \oplus \cdots K_{t}^{\prime}$ is a semi-simple Lie algebra with $K_{j}{ }^{\prime}=K_{j}{ }^{\prime} K_{j}{ }^{\prime}$ a simple ideal, then for a derivation $D$ of $K^{\prime}$ we have $D K_{j}{ }^{\prime}=$ $D\left(K_{j}{ }^{\prime} K_{j}{ }^{\prime}\right)=\left(D K_{j}{ }^{\prime}\right) K_{j}{ }^{\prime}+K_{j}{ }^{\prime}\left(D K_{j}{ }^{\prime}\right) \subset K_{j}{ }^{\prime}$. Thus since the above spaces in the decomposition are all $D(\mathfrak{h})$-invariant we have

$$
[D(\mathfrak{h}), S]=0 \text { and } S^{b}=S
$$

where $b$ denotes the adjoint relative to $B(X, Y)=\operatorname{Kill}(X, Y)$. Thus by (2. 9) we can define an algebra $(\mathfrak{m}, \alpha)$ which gives a pseudo-Riemannian connection relative to $C(X, Y)=\operatorname{Kill}(S X, Y)$. We compute the multiplication for $(\mathfrak{m}, \alpha)$ relative to the above decomposition for $\mathfrak{m}$.

Let $A, B \in c, P_{j}, Q_{j} \in K_{j}{ }^{\prime}, U, V \in K_{1}, X, Y \in K_{2}$, then

$$
\begin{array}{ll}
\alpha(A, B)=0 & \alpha\left(A, P_{j}\right)=0 \\
\alpha(A, U)=0 & 2 \alpha(A, X)=\frac{2 s_{4}-s_{1}}{s_{4}} A X \in K_{2} \\
\alpha\left(P_{j}, A\right)=0 & 2 \alpha\left(P_{j}, Q_{i}\right)=P_{j} Q_{i} \in K_{j}{ }^{\prime} \cap K_{i}{ }^{\prime} \\
\alpha\left(P_{j}, U\right)=0 & 2 \alpha\left(P_{j}, X\right)=\frac{2 s_{4}-s_{2 j}}{s_{4}} P_{j} X \in K_{2} \\
\alpha(U, A)=0 & \alpha\left(U, P_{j}\right)=0 \\
2 \alpha(U, V)=U V \in K_{1} & 2 \alpha(U, X)=\frac{2 s_{4}-s_{3}}{s_{4}} U X \in K_{2}
\end{array}
$$




$$
\begin{array}{ll}
2 \alpha(X, A)=-\frac{s_{1}}{s_{4}} A X \in K_{2} & 2 \alpha\left(X, P_{j}\right)=-\frac{s_{2 j}}{s_{4}} P_{j} X \in K_{2}^{\prime} \\
2 \alpha(X, U)=-\frac{s_{3}}{s_{4}} U X \in K_{2} & 2 \alpha(X, Y)=X Y \in \mathfrak{m} .
\end{array}
$$

Proposition 3. Let $(\mathfrak{g}, \mathfrak{h})$ be a nonsymmetric reductive pair with $\mathfrak{g}$ simple, $\mathfrak{h}$ semi-simple and $\mathfrak{g}=\mathfrak{m}+\mathfrak{h}$ with $\mathfrak{m}=\mathfrak{h} \perp$ relative to the Killing form. Let $\mathfrak{m}=c \dot{+} K^{\prime}+K_{1}+K_{2}$ be the decomposition previously discussed and let $S: \mathfrak{m} \rightarrow \mathfrak{m}$ be as above where $2 s_{4}-s_{1} \neq 0,2 s_{4}-s_{2 j} \neq 0$ and $2 s_{4}-s_{3} \neq 0$. Then the algebra $(\mathfrak{m}, \alpha)$ determined by $S$ has no proper $D(\mathfrak{h})$-invariant left ideals. Thus by [8] if the corresponding space $G / H$ is simple connected, then $G / H$ is holonomy irreducible relative to the pseudo-Riemannian connection induced by $C(X, Y)=\operatorname{Kill}(S X, Y)$ and the algebra $(\mathfrak{m}, \alpha)$.

Proof. Let $\mathfrak{i t}$ be a proper $D(\mathfrak{h})$-invariant left ideal of ( $\mathfrak{m}, \alpha)$ and let $\mathfrak{n}(c), \mathfrak{n}^{\prime}(j), \mathfrak{n}(1)$ and $\mathfrak{n}(2)$ be the projection of $\mathfrak{n}$ into $c, K_{j}^{\prime}, K_{1}$ and $K_{2}$ respectively. Thus, for example, $\mathfrak{i t}(c)$ is the set of $B \in c$ such that there exist $Q_{j} \in K_{j}{ }^{\prime}, V \in K_{1}, Y \in K_{2}$ with $B+\Sigma Q_{j}+V+Y \in \mathfrak{H}$. We shall show that all the subspaces $($ of $\mathfrak{m}) \mathfrak{n}(c), \cdots, \mathfrak{n}(2)$ are in $\mathfrak{n}$ and use this to show $(\mathfrak{n}, X Y$ ) is a proper ideal of $(\mathfrak{m}, X Y)$ which contradicts the results in Proposition 2 (b).

First we note that the subspaces $\mathfrak{t}(c), \mathfrak{H}^{\prime}(j), \mathfrak{t}(1), \mathfrak{t}(2)$ are all $D(\mathfrak{h})$-invariant since $\mathfrak{n}$ is $D(\mathfrak{h})$-invariant. Next we shall show

$$
D(\mathfrak{h}) \mathfrak{t}(i)=\mathfrak{t}(i) \text { for } i=1,2 \text { and } \mathfrak{n t}(2)=L(K) \mathfrak{t}(2) \subset \mathfrak{t} \text {. }
$$

For suppose $D(\mathfrak{h}) \mathfrak{n}(1) \subset \mathfrak{n}(1)$ properly, then $p=D(\mathfrak{h}) \mathfrak{n}(1)$ is a proper $D(\mathfrak{h})$ submodule. Since $D(\mathfrak{h})$ is completely reducible in $\mathfrak{n}(1)$ we have $\mathfrak{n}(1)=p \dot{+} p^{\prime}$ where $p^{\prime}$ is a $D(\mathfrak{h})$-invariant complement. Thus $D(\mathfrak{h}) p^{\prime} \subset p^{\prime} \cap D(\mathfrak{h}) \mathfrak{n}(1)=p^{\prime} \cap p=0$; that is, $p^{\prime} \subset K$ which is a contradicition unless $p^{\prime}=0$. This gives $\mathfrak{n t}(1)=p=$ $D(\mathfrak{h}) \mathfrak{n}(1)$ and similarly for $\mathfrak{t}(2)$. Next from Proposition 2 (d) we see that $L(K) \cong a d(K)$ maps $K_{2}$ onto $K_{2}$ and is completely reducible in $K_{2}$. From the preceding let $N_{2}=\sum_{i} D_{i} N_{i 2} \in \mathfrak{n}(2)=D(\mathfrak{h}) \mathfrak{n}(2)$ where the $N_{i 2} \in \mathfrak{H}(2)$, then we can find elements $N_{0 i} \in \mathfrak{n}(c), N_{j_{i}}^{\prime} \in \mathfrak{n}^{\prime}(j)$ and $N_{i 1} \in \mathfrak{n}(1)$ such that $q_{i}=N_{0 i}+$ $\sum_{j} N_{j 2}^{\prime}+N_{i 1}+N_{i 2} \in \mathfrak{t}$. But then $q=\sum D_{i} q_{i}=\sum D_{i} N_{i 1}+\sum D_{i} N_{i 2}=\bar{N}_{1}+N_{2} \in \mathfrak{H}$ where $\bar{N}_{1} \in \mathfrak{n}(1)$. Thus for any $A \in c$ and $P_{j} \in K_{j}^{\prime}$ we have

$$
2 \alpha(A, q)=\frac{2 s_{4}-s_{1}}{s_{4}} A N_{2} \in \mathfrak{H} \cap K_{2} \text { and }
$$




$$
2 \alpha\left(P_{j}, q\right)=\frac{2 s_{4}-s_{2 j}}{s_{4}} P_{j} N_{2} \in \mathfrak{i} \cap K_{2} .
$$

Using the hypothesis, these imply $L(K) \mathfrak{n}(2) \subset \mathfrak{n} \cap K_{2} \subset \mathfrak{n}(2)$ and of course $L(K) \mathfrak{n}(2) \subset \mathfrak{n}$. Thus we shall now show $\mathfrak{n}(2)=L(K) \mathfrak{n}(2)$. For $\mathfrak{n}(2) \neq 0$ implies $p=L(K) \mathfrak{t}(2)$ is a nonzero $L(K)$-submodule of $\mathfrak{n}(2)$ and if $p$ is a proper submodule, then since $L(K)$ is completely reducible in $\mathfrak{n}(2)$ we can write $\mathfrak{n}(2)=p+p^{\prime}$ where $p^{\prime}$ is $L(K)$-invariant. Thus $L(K) p^{\prime} \subset p^{\prime} \cap L(K) \mathfrak{n}(2)=$ $p^{\prime} \cap p=0$ so that $p^{\prime} \subset \mathfrak{n}(1)$ i.e. $p^{\prime}=0$ and $\mathfrak{n t}(2)=L(K) \mathfrak{t}(2)$.

Next let $N_{1}=\sum D_{i} N_{i 1} \in \mathfrak{n}(1)=D(h) \mathfrak{n}(1)$ where $N_{i 1} \in \mathfrak{n}(1)$ and let $N_{0 i} \in \mathfrak{n}(c)$, $N_{j i}^{\prime} \in \mathfrak{t}^{\prime}(j)$ and $N_{i 2} \in \mathfrak{n}(2)$ be such that $p_{i}=N_{0 i}+\sum_{j} N_{j i}^{\prime}+N_{i 1}+N_{i 2} \in \mathfrak{l t}$. Then $p=\sum D_{i} p_{i}=\sum D_{i} N_{i 1}+\sum D_{i} N_{i 2}=N_{1}+\bar{N}_{2} \in \mathfrak{l t}$ where $\bar{N}_{2} \in \mathfrak{n}(2)$. But $\mathfrak{n}(2) \subset \mathfrak{l}$ yields $N_{1}=p-\bar{N}_{2} \in \mathfrak{n}$ i.e. $\mathfrak{n}(1) \subset \mathfrak{n}$. Note if $K=0, K_{2}=0$ so just use the above argument.

Finally let $N_{j}^{\prime} \in \mathfrak{t t}^{\prime}(j) \neq 0$, then since $\mathfrak{t}(1)$ and $\mathfrak{t}(2)$ are in $\mathfrak{t t}$, we can find $N_{0} \in \mathfrak{n}(c)$ and $N_{i}^{\prime} \in \mathfrak{n}^{\prime}(i)$ with $i \neq j$ so that $r=N_{0}+N_{j}^{\prime}+\sum N_{i}^{\prime} \in \mathfrak{l t}$. Then $2 \alpha\left(P_{j}, r\right)=P_{j} N_{j}^{\prime} \in \mathfrak{t} \cap K_{j}^{\prime} \subset \mathfrak{t}^{\prime}(j)$, which shows $\mathfrak{t}^{\prime}(j)$ is a nonzero ideal of the simple Lie algebra $K_{j}^{\prime}$. Thus $K_{\jmath}^{\prime}=\mathfrak{t}^{\prime}(j)$ and using the preceding computation we obtain since $K_{j}{ }^{\prime}=K_{j}{ }^{\prime} K_{j}{ }^{\prime}$

$$
\mathfrak{n}^{\prime}(j)=K_{j}^{\prime} \mathfrak{n}^{\prime}(j) \subset \mathfrak{n} \cap K_{j}^{\prime} \subset \mathfrak{l} .
$$

Therefore since $\mathfrak{n}^{\prime}(j), \mathfrak{n}(1)$ and $\mathfrak{n}(2)$ are in $\mathfrak{n}$ so is $\mathfrak{n}(c)$. We use this to obtain $\mathfrak{n}=\mathfrak{n}(c)+\sum \mathfrak{h}^{\prime}(j)+\mathfrak{n}(1)+\mathfrak{n}(2)$ as a subspace decomposition, the multiplicative relations preceding Proposition 3 , and the hypothesis to obtain $\mathfrak{m} \mathfrak{t} \subset \alpha(\mathfrak{m}, \mathfrak{t}) \subset \mathfrak{n}$. Thus $\mathfrak{n}$ is proper ideal of ( $\mathfrak{n}, X Y)$, a contradiction.

Remark. Starting with the pair $(G, H)$ where $G$ is a connected Lie group and $H$ is a closed Lie subgroup, we obtain the reductive pair of Lie algebras $(\mathfrak{g}, \mathfrak{h})$ so that conditions on the algebras give algebraic results on connections. But to easily translate these conditions-in particular, holonomy irreducibility-back to $M=G / H$ we need that $M$ is simple connected. If this is not the case, we shall construct a connected Lie group $\widetilde{G}$ with Lie algebra $g$ and a closed connected subgroup $\tilde{H}$ with Lie algebra $\mathfrak{h}$ so that $\tilde{M}=\tilde{G} / \tilde{H}$ is simple connected. Furthermore, since the pair $(\tilde{G}, \tilde{H})$ yields the corresponding reductive pair of Lie algebras $(\mathfrak{g}, \mathfrak{h}), \tilde{M}$ is a reductive homogeneous space to which the algebraic conditions can be applied. Thus let $\widetilde{G}$ be the connected simply connected universal covering Lie group of $G$ and 
let $\phi: \widetilde{G} \rightarrow G$ be the corresponding covering epimorphism. Then $\widetilde{G}$ has Lie algebra $g$ and $\tilde{K}=\phi^{-1}(H)$ is a closed Lie subgroup of $\tilde{G}$ and the Lie algebra of $\tilde{K}$ is $\mathfrak{h}$, using $\phi$ as a local isomorphism. Now let $\tilde{H}=(\tilde{K})_{0}=$ the connected component of $\tilde{K}$ in $\tilde{K}$. Then $\tilde{H}$ is closed in $\tilde{K}$ and since $\tilde{K}$ is closed in $\widetilde{G}, \tilde{H}$ is closed in $\tilde{G}$. Thus since the Lie algebra of $\tilde{H}$ is $\mathfrak{h}$, the pair $(\tilde{G}, \tilde{H})$ has the corresponding reductive pair of Lie algebras $(\mathfrak{g}, \mathfrak{h})$ so that $\tilde{M}=\tilde{G} / \tilde{H}$ is a reductive homogeneous space.

Next $\tilde{M}$ is simply connected. First, $\tilde{M}$ is connected since it is the image of the continuous map $\tilde{\pi}: \tilde{G} \rightarrow \tilde{G} / \tilde{H}$ of the connected space $\tilde{G}$. Thus since $\tilde{M}$ is a connected manifold, it is also path-connected. Now regard $\tilde{G} / \tilde{H}$ as a fiber bundle where the total space is $E=\widetilde{G}$, the base space is $B=\tilde{G} / \tilde{H}$, the fiber is $F=\tilde{H}$, and the projection is $\tilde{\pi}: \tilde{G} \rightarrow \tilde{G} / \tilde{H}$. Then from the last few terms of the homotopy exact sequence of a fiber map extended to $\pi_{0}[1$, p. $8 ; 9$, p. 377] we have for the identity $\tilde{e}$ of $\tilde{G}$,

$$
\rightarrow \pi_{1}(\tilde{G}, \tilde{e}) \stackrel{\tilde{\pi}_{*}}{\longrightarrow} \pi_{1}(\tilde{G} / \tilde{H}, \tilde{H}) \stackrel{\partial}{\longrightarrow} \pi_{0}(\tilde{H}, \tilde{e}) \rightarrow .
$$

But since $\tilde{G}$ is simply connected, $\pi_{1}(\tilde{G}, \tilde{e})=0$ and since $\tilde{H}$ is connected, $\pi_{0}(\tilde{H}, \tilde{e})=0$. Thus by the exactness of the sequences,

$$
0=\operatorname{Im} \tilde{\pi}_{*}=\operatorname{Ker} \partial=\pi_{1}(\tilde{G} / \tilde{H}, \tilde{H}) .
$$

Thus since $\tilde{M}$ is path-connected, it is simply connected.

Next using (3. 3) we shall derive the equations so that the connection discussed in Proposition 3 makes $G / H$ into an Einstein manifold; that is, we have an Einstein connection. First we compute the matrices for the left multiplications $L(Z)$ for $Z \in \mathfrak{m}$. From Proposition 2 (b) we have for $A, B \in c$

$$
L(A)=\left(\begin{array}{llll}
0 & 0 & 0 & 0 \\
0 & 0 & 0 & 0 \\
0 & 0 & 0 & 0 \\
0 & 0 & 0 & A_{44}
\end{array}\right)
$$

and $\operatorname{Kill}(A, B)=\operatorname{tr} L(A) L(B) . \quad$ For $P_{j}, Q_{j} \in K_{j}$

$$
L\left(P_{j}\right)=\left(\begin{array}{llll}
0 & 0 & 0 & 0 \\
0 & P_{22} & 0 & 0 \\
0 & 0 & 0 & 0 \\
0 & 0 & 0 & P_{44}
\end{array}\right)
$$


and $\operatorname{Kill}\left(P_{j}, Q_{j}\right)=\operatorname{tr} L\left(P_{j}\right) L\left(Q_{j}\right) . \quad$ For $U, V \in K_{1}$

$$
L(U)=\left(\begin{array}{llll}
0 & 0 & 0 & 0 \\
0 & 0 & 0 & 0 \\
0 & 0 & U_{33} & 0 \\
0 & 0 & 0 & U_{44}
\end{array}\right)
$$

and $\operatorname{Kill}(U, V)=\operatorname{tr} L(U) L(V)+2 \operatorname{tr} \sigma(U, V)$. For $X, Y \in K_{2}$

$$
L(X)=\left(\begin{array}{cccc}
0 & 0 & 0 & X_{14} \\
0 & 0 & 0 & X_{24} \\
0 & 0 & 0 & X_{34} \\
X_{41} & X_{42} & X_{43} & X_{44}
\end{array}\right)
$$

and $\operatorname{Kill}(X, Y)=\operatorname{tr} L(X) L(Y)+2 \operatorname{tr} \sigma(X, Y)$. Note that the matrices $P_{22}, X_{24}$ and $X_{42}$ can be put into block form according to the decomposition $K^{\prime}=K_{1}^{\prime} \oplus \cdots \oplus K^{\prime}{ }_{t}$.

Now we note that the orthogonality of $c, K^{\prime}, K_{1}$ and $K_{2}$ relative to the Killing form give corresponding solutions to $(3.3)$ as follows.

$$
\begin{aligned}
f\left(A, P_{j}, S, \eta\right)= & \left(\eta S_{1}-2\right) \operatorname{Kill}\left(A, P_{j}\right)+2 \operatorname{tr} L(A) S^{-1} L\left(P_{j}\right) S \\
& \quad-s_{1} s_{2 j} \operatorname{tr} L(A) S^{-1} L\left(P_{j}\right) S^{-1} \\
= & 0
\end{aligned}
$$

using the block matrices for $L(A)$ and $L\left(P_{j}\right)$ and $0=\operatorname{Kill}\left(A, P_{j}\right)=\operatorname{tr} A_{44} P_{44}$. Similarly $f(A, U, S, \eta)=f(A, X, S, \eta)=0$. Also for $i \neq j, f\left(P_{j}, Q_{i}, S, \eta\right)=$ $f\left(P_{j}, U, S, \eta\right)=f\left(P_{j}, X, S, \eta\right)=f(U, X, S, \eta)=0$. For the last equation we must also use

$$
\operatorname{tr} \sigma\left(K_{1}, K_{2}\right)=0
$$

which yields $0=\operatorname{Kill}(U, X)=\operatorname{tr} L(U) L(X)+2 \operatorname{tr} \sigma(U, X)=\operatorname{tr} L(U) L(X)=\operatorname{tr} U_{44} X_{44}$. To see $\operatorname{tr} \sigma\left(K_{1}, K_{2}\right)=0$ we note from Proposition 2 (d) that $D\left(K_{1}, K_{2}\right)=0$. Thus $\quad \sigma\left(K_{1}, K_{2}\right) c=D\left(c, K_{2}\right) K_{1}=0 ; \quad \sigma\left(K_{1}, K_{2}\right) K^{\prime}=D\left(K^{\prime}, K_{2}\right) K_{1}=0 ; \quad \sigma\left(K_{1}, K_{2}\right) K_{1}=$ $D\left(K_{1}, K_{2}\right) K_{1}=0 ; \sigma\left(K_{1}, K_{2}\right) K_{2}=D\left(K_{2}, K_{2}\right) K_{1} \subset K_{1}$ which yields $\operatorname{tr} \sigma\left(K_{1}, K_{2}\right)=0$.

Next we consider the nonorthogonal equations. For $A, B \in c$ we have

$$
\begin{gathered}
f(A, B, S, \eta)=\left(\eta s_{1}-2\right) \operatorname{Kill}(A, B)+2 \operatorname{tr} L(A) S^{-1} L(B) S \\
-s_{1}{ }^{2} \operatorname{tr} L(A) S^{-1} L(B) S^{-1}
\end{gathered}
$$




$$
\begin{aligned}
= & \left(\eta s_{1}-2\right) \operatorname{tr} A_{44} B_{44}+2 \operatorname{tr} A_{44} B_{44} \\
& \quad-s_{1}{ }^{2}\left(s_{4}{ }^{-2} \operatorname{tr} A_{44} B_{44}\right) \\
= & s_{1}\left(\eta-s_{1} s_{4}{ }^{-2}\right) \operatorname{tr} A_{44} B_{44}
\end{aligned}
$$

and since $\operatorname{Kill}(A, B)=\operatorname{tr} A_{44} B_{44}$ is nondegenerate on $c$ we must have

$$
\eta=s_{1} s_{4}^{-2}
$$

For $P_{j}, Q_{j} \in K_{j}^{\prime}$ we have

$$
\begin{aligned}
& f\left(P_{j}, Q_{j}, S, \eta\right)=\left(\eta s_{2 j}-2\right) \operatorname{Kill}\left(P_{j}, Q_{j}\right) \\
& +2 \operatorname{tr} L\left(P_{j}\right) S^{-1} L\left(Q_{j}\right) S \\
& \text { - }\left(s_{2 j}\right)^{2} \operatorname{tr} L\left(P_{j}\right) S^{-1} L\left(Q_{j}\right) S^{-1} \\
& =\left(\eta s_{2 j}-2\right) \operatorname{tr} L\left(P_{j}\right) L\left(Q_{j}\right) \\
& +2 \operatorname{tr}\left(\begin{array}{llll}
0 & & & \\
& P_{22} s_{2 j}-1 & \\
& & 0 & \\
& & P_{44} s_{4}{ }^{-1}
\end{array}\right)\left(\begin{array}{llll}
0 & & & \\
& Q_{22} s_{2 j} & & \\
& & 0 & \\
& & & Q_{44} s_{4}
\end{array}\right)
\end{aligned}
$$

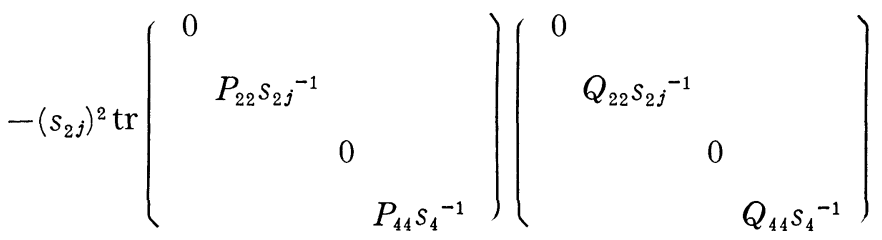

$$
\begin{aligned}
& =\left(\eta s_{2 j}-1\right) \operatorname{tr} P_{22} Q_{22}+\left(\eta s_{2}-s_{2 j}{ }^{2} s_{4}^{-2}\right) \operatorname{tr} P_{44} Q_{44} \\
& =\left(\eta s_{2 j}-1\right) \operatorname{Kill}_{j}\left(P_{j}, Q_{j}\right)+s_{2 j}\left(\eta-s_{2 j} s_{4}{ }^{-2}\right) \operatorname{tr} \rho\left(P_{j}\right) \rho\left(Q_{j}\right)
\end{aligned}
$$

where $\mathrm{Kill}_{j}$ denotes the Killing form of the simple Lie algebra $K_{j}{ }^{\prime}$ and $\rho: K_{j}{ }^{\prime} \rightarrow \operatorname{Hom}\left(K_{2}, K_{2}\right): P_{j} \rightarrow \rho\left(P_{j}\right)$ denotes the representation of $K_{j}^{\prime}$ given by $\rho\left(P_{j}\right) X=P_{j} X$. But since $\operatorname{tr} \rho\left(P_{j}\right) \rho\left(Q_{j}\right)$ is an invariant form i.e. $B(X Y, Z)=$ $B(X, Y Z)$, there exists a self-adjoint linear transformation $T=T_{j} \in \operatorname{Hom}\left(K_{j}{ }^{\prime}, K_{j}{ }^{\prime}\right)$ with $\operatorname{tr} \rho\left(P_{j}\right) \rho\left(Q_{j}\right)=\operatorname{Kill}_{j}\left(T P_{j}, Q_{j}\right)$ and $T$ is in the centroid $\Gamma\left(K_{j}^{\prime}\right)$, see [2, p. $290 ; 7]$. To see this we note

$$
\begin{aligned}
\operatorname{Kill}_{j}\left(T L\left(P_{j}\right) Q_{j}, R_{j}\right) & =\operatorname{tr} \rho\left(P_{j} Q_{j}\right) \rho\left(R_{j}\right) \\
& =\operatorname{tr} \rho\left(P_{j}\right) \rho\left(Q_{j} R_{j}\right) \\
& =\operatorname{Kill}_{j}\left(T P_{j}, Q_{j} R_{j}\right) \\
& =\operatorname{Kill}_{j}\left(L\left(T P_{j}\right) Q_{j}, R_{j}\right)
\end{aligned}
$$


so that $T L\left(P_{j}\right)=L\left(T P_{j}\right)$. This yields $\left[T, L\left(P_{j}\right)\right]=0$ and therefore $T \in \Gamma\left(K_{j}{ }^{\prime}\right)$. But $\Gamma\left(K_{j}{ }^{\prime}\right)$ is a field which is at worst isomorphic to the complex numbers [2]. Thus $T=a_{j} I+b_{j} J$ where $J^{2}+I=0$ and $a, b$ are real. Combining this with the equation involving $S$ we must have

$$
\left.\mathrm{Kill}_{j}\left(\left[\eta s_{2 j}-1\right) I+s_{2 j}\left(\eta-s_{2 j} s_{4}{ }^{-2}\right) T\right] P_{j}, Q_{j}\right)=0
$$

which yields

$$
\begin{gathered}
\left(\eta s_{2 j}-1\right)+a_{j} s_{2 j}\left(\eta-s_{2 j} S_{4}^{-2}\right)=0 \quad \text { and } \\
b_{j} s_{2 j}\left(\eta-s_{2 j} s_{4}^{-2}\right)=0 .
\end{gathered}
$$

In particular if $\operatorname{Kill}_{j}\left(P_{j}, Q_{j}\right)$ or $\operatorname{Kill}_{g}\left(P_{j}, Q_{j}\right)$ or $\operatorname{tr} \rho\left(P_{j}\right) \rho\left(Q_{j}\right)$ are definite (positive or negative), then $T_{j}$ has a real eigenvalue which implies $b_{j}=0$. For $U, V \in K_{1}$ we must have

$$
\begin{aligned}
& f(U, V, S, \eta)=\left(\eta s_{3}-2\right) \operatorname{Kill}(U, V)+
\end{aligned}
$$

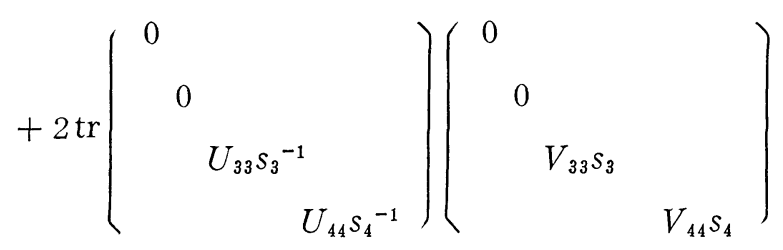

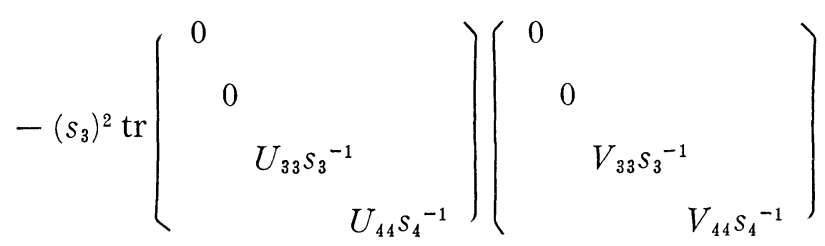

$$
\begin{aligned}
& =2\left(\eta s_{3}-2\right) \operatorname{tr} \sigma(U, V)+\left(\eta s_{3}-1\right) \operatorname{tr} U_{33} V_{33} \\
& \text { (3. 11) } \quad+\left(\eta s_{3}-s_{3}{ }^{2} s_{4}{ }^{-2}\right) \operatorname{tr} U_{44} V_{44} \\
& =0 \text {. }
\end{aligned}
$$

Finally for $X, Y \in K_{2}$ we first note that in the matrix (3.8) for $L(X)$ the blocks $X_{42}$ and $X_{24}$ are block matrices since $K^{\prime}=K_{1}^{\prime} \oplus \cdots \oplus K_{t}^{\prime}$. Thus write $X_{42}=\left[X_{42}(1) \cdots X_{42}(t)\right]$ and similarly for $X_{24}$. Now we must have

$$
\begin{aligned}
f(X, Y, S, \eta)= & \left(\eta s_{4}-2\right) \operatorname{Kill}(X, Y)+2 \operatorname{tr} L(X) S^{-1} L(Y) S \\
& \quad-\left(s_{4}\right)^{2} \operatorname{tr} L(X) S^{-} L(Y) S^{-1} \\
= & 2\left(\eta s_{4}-2\right) \operatorname{tr} \sigma(X, Y) \\
& \quad+\left(\eta s_{4}-2+2 s_{1} s_{4}^{-1}-s_{4} s_{1}^{-1}\right) \operatorname{tr} X_{14} Y_{41}
\end{aligned}
$$




$$
\begin{aligned}
& +\left(\eta s_{4}{ }^{-2}+s_{1}{ }^{-} s_{4}\right) \operatorname{tr} X_{41} Y_{14} \\
& +\left(\eta s_{4}-2+2 s_{3} s_{4}^{-1}-s_{4} s_{3}^{-1}\right) \operatorname{tr} X_{34} Y_{43} \\
& +\left(\eta s_{4}^{-2}+s_{4} s_{3}{ }^{-1}\right) \operatorname{tr} X_{43} Y_{34}+\left(\eta s_{4}-1\right) \operatorname{tr} X_{44} Y_{44} \\
& +\sum_{j=1}^{t}\left(\eta s_{4}-2+2 s_{4}^{-1} s_{2 j}-s_{4} s_{2 j}^{-1}\right) \operatorname{tr} X_{24}(j) Y_{42}(j) \\
& +\sum_{j=1}^{t}\left(\eta s_{4}-2+s_{4} s_{2 j}^{-1}\right) \operatorname{tr} X_{42}(j) Y_{24}(j) \\
& =0
\end{aligned}
$$

Remarks. (1) In the next section we shall make various irreducibility assumptions on the spaces $K_{1}$ and $K_{2}$ which makes the computation of the "trace forms" in (3.11) and (3. 12) more reasonable.

(2) The classification of holonomy irreducible Einstein manifolds relative to the connection of the first kind (i.e. $S=\lambda I$ ) appears to be an open problem. Some progress has been made for low dimensions by $G$. Hensen in his thesis at the University of California at Berkely. Also if $\mathfrak{h}$ acts irreducibly on $\mathfrak{m}$, then one obtains an Einstein connection [11]. For in this case let $B(X, Y)$ be a metric inducing the connection, then there exists $S \in \mathfrak{A}$ with $\operatorname{Ric}(X, Y)=B(S X ; Y)$ where $\mathfrak{A}$ is the Jordan algebra previously discussed. But since $B(X, Y)$ is positive definite, $S$ is symmetric and has a real eigenvalue $\lambda$. Thus if $\operatorname{Ric}(X, Y) \not \equiv 0$, then $\{X \in \mathfrak{m}: S X=\lambda X\}$ is a nonzero $\mathfrak{h}$-invariant subspace of $\mathfrak{m}$ which therefore must equal $\mathfrak{m}$. Thus $S=\lambda I$ so $B(X, Y)=\frac{1}{\lambda} \operatorname{Ric}(X, Y)$.

In the next section we shall give an easy test to see if a given reductive pair $(\mathfrak{g}, \mathfrak{h})$ induces an Einstein connection of the first kind.

\section{Some examples.}

We now compute some general examples which yield holonomy irreducible Einstein manifolds as previously discussed. Thus using the decomposition $\mathfrak{m}=c+K^{\prime}+K_{1}+K_{2}$ we shall show that the corresponding $S: \mathfrak{m} \rightarrow \mathfrak{m}$ exists i.e. the equations (3. 9)-(3.12) have solutions. Then we shall note that $S$ can be chosen to satisfy Proposition 3 i.e. the algebra ( $\mathfrak{n}, \alpha$ ) has no $\mathfrak{h}$ invariant left ideals.

For the first example let $g$ be the $n \times n$ real skew-symmetric matrices $\mathfrak{g} \mathfrak{D}(n)$ and let $\mathfrak{h}$ be the $k \times k$ real skew-symmetric matrices where $3 \leq k<n-2$; thus we are essentially considering the algebra of the non-symmetric Stiefel 
manifold $S O(n) / S O(k)$ which is simply connected [1, p. 83]. We let $\mathrm{g}$ be represented by the skew-symmetric matrices

$$
\left[\begin{array}{ll}
F_{11} & F_{12} \\
-F_{12}{ }^{t} & h_{22}
\end{array}\right]
$$

where the $h_{22}$ is $k \times k$ skew-symmetric. We identify $h$ with the set

$$
\left[\begin{array}{ll}
0 & 0 \\
0 & h_{22}
\end{array}\right]
$$

and note that $\mathfrak{m}=\mathfrak{h} \perp$ equals the set

$$
\left[\begin{array}{ll}
F_{11} & F_{12} \\
-F_{12}{ }^{t} & 0
\end{array}\right] .
$$

Next let $n=k+r$ so that $K$ is identified with the $r \times r$ skew-symmetric matrices where $r \geq 3$. Thus $K=K^{\prime}$ is simple except when $r=4$. In this case $K^{\prime}=K_{1}^{\prime} \oplus K_{2}^{\prime}$ and we let $s_{21}=s_{22}=s_{2}$. Thus $K$ equals the set

$$
\left[\begin{array}{ll}
F_{11} & 0 \\
0 & 0
\end{array}\right]
$$

Furthermore, $K_{1}=0$ so that $b=K \perp=K_{2}$ which equals the set

$$
\left[\begin{array}{ll}
0 & F_{12} \\
-F_{12}{ }^{t} & 0
\end{array}\right] \text {. }
$$

Now by Proposition $2(\mathrm{~d}), a d_{g}(\mathfrak{h}+K)$ acts on $b$ and this action is irreducible. This can be seen by noting $a d(\mathfrak{h}+K)$ is isomorphic to $\mathfrak{g} \mathfrak{D}(k)+\mathfrak{g}_{\mathfrak{D}}(r)$ where $k+r>4$ and the symmetric pair $(\mathfrak{g} \mathfrak{D}(n), \quad \mathfrak{g} \mathfrak{D}(k)+\mathfrak{g} \mathfrak{D}(r))$ is irreducible. This means that in the decomposition $\mathfrak{g}=\mathfrak{h}+K \dot{+} b a d(\mathfrak{h}+K)$ acts irreducibly on $b$; this can also be shown by straight forward matrix computations.

We now determine $S$ relative to the decomposition $\mathfrak{m}=K^{\prime}+K_{2}$ and use the various irreducibility conditions to help solve equations (3.10) and (3. 12).

A straight forward computation shows that we always have for $P \in K^{\prime}$

$$
\operatorname{tr} \rho(P) \rho(P)=\frac{k}{r-2} \mathrm{Kill}_{K^{\prime}}(P, P)
$$

so $a=k / r-2$ and (3. 10) becomes 


$$
(r-2)\left(\eta s_{2}-1\right)+k s_{2}\left(\eta-s_{2} s_{4}^{-2}\right)=0
$$

where $k+r=n$. Next equation (3.12) becomes

$$
\begin{aligned}
& 2\left(\eta s_{4}-2\right) \operatorname{tr} \sigma(X, Y)+\left(\eta s_{4}-1\right) \operatorname{tr} X_{44} Y_{44} \\
& +\left(\eta s_{4}-2+2 s_{4}^{-1} s_{2}-s_{4} s_{2}^{-1}\right) \operatorname{tr} X_{24} Y_{42}+\left(\eta s_{4}-2+s_{4} s_{2}^{-1}\right) \operatorname{tr} X_{42} Y_{24} \\
& =0
\end{aligned}
$$

noting $X_{14}=X_{34}=0$ in (3. 8). We can further simplify as follows. First note that $\left[K_{2}, K_{2}\right] \subset K+\mathfrak{h}$ so that $K_{2} K_{2} \subset K$ which gives $X_{44}=0$. Next note that $L(X)$ is skew-symmetric so that $X_{24}=-X_{42}{ }^{\prime} ;$ thus we obtain $\operatorname{tr} L(X) L(Y)$ $=-2 \operatorname{tr} X_{24} Y_{24}{ }^{\prime}$ and consequently

$$
2\left(\eta s_{4}-2\right) \operatorname{tr} \sigma(X, Y)+\left(\eta s_{4}-2+s_{2} s_{4}{ }^{-1}\right) \operatorname{tr} L(X) L(Y)=0 .
$$

Next we use the $a d(\mathfrak{h}+K)$-irreducibility of $K_{2}=b$ as follows. Since $D(K, \mathfrak{m})=0$, we see that $a d(\mathfrak{h} \dot{+}$ ) acts like derivations on the algebra $(\mathfrak{m}, X Y)$ by Proposition 2 (d). Thus for $D \in a d(\mathfrak{h}+K)$ we have

$$
L(D X)=[D, L(X)]
$$

which yields $\operatorname{tr} L(D X) L(Y)=-\operatorname{tr} L(X) L(D Y)$. Thus the form $\operatorname{tr} L(X) L(Y)$ is $a d(\mathfrak{h}+K)$-invariant. Since $\operatorname{Kill}(X, Y)$ is also $a d(\mathfrak{h}+K)$-invariant and $\operatorname{Kill}(X, Y)=\operatorname{tr} L(X) L(Y)+2 \operatorname{tr} \sigma(X, Y)$ we have $\operatorname{tr} \sigma(X, Y)$ is $a d(\mathfrak{G}+K)$-invariant. Using this we can write

$$
\begin{aligned}
& \operatorname{tr} L(X) L(Y)=\operatorname{Kill}\left(T_{1} X, Y\right) \\
& \operatorname{tr} \sigma(X, Y)=\operatorname{Kill}\left(T_{2} X, Y\right)
\end{aligned}
$$

where $T_{i}$ are self-adjoint relative to $\operatorname{Kill}(X, Y)$ and $\left[T_{i}, a d(\mathfrak{h} \dot{+} K)\right]=0$. Thus since $\operatorname{Kill}(X, Y)$ is positive definite the $T_{i}$ have eigenvalues. But since $K_{2}$ is $\operatorname{ad}(\mathfrak{h}+K)$-irreducible this gives $T_{i}=b_{i} I$. Thus from (4.2) we obtain

$$
b_{1}\left(\eta s_{4}-2+s_{2} s_{4}^{-1}\right)+2 b_{2}\left(\eta s_{4}-2\right)=0
$$

where a straightforward calculation shows

$$
b_{1}=(r-1) /(n-2) \text { and } b_{2}=(k-1) / 2(n-2) .
$$

Thus after simplification we must solve the two equations

$$
\begin{aligned}
& (r-2) u^{2}+k v^{2}-(n-2) u^{2} v=0 \\
& (n-2) u^{2}-2(n-2) u+(r-1) v=0
\end{aligned}
$$


where $k+r=n, u=\eta s_{4}, v=\eta s_{2}$. From (4. 5) we obtain

$$
v=\left(\frac{n-2}{r-1}\right)(2-u) u
$$

which substituted into (4. 4) gives

$$
\begin{aligned}
& (n-2)^{2}(n-1) u^{2}-2(n-2)^{2}(n+k-1) u \\
& +4 k(n-2)^{2}+(r-2)(r-1)^{2}=0
\end{aligned}
$$

and a solution exists. Thus a mapping $S$ exists which yields an Einstein space which is furthermore irreducible. For this last we must only show $2 s_{4} \neq s_{2}$. Thus assume $2 u=v$, then substituting into (4. 4) we obtain

$$
u=\frac{n+3 k-2}{2(n-2)} .
$$

But substituting $2 u=v$ into (4.5) we obtain

$$
u=\frac{2(k-1)}{n-2}
$$

which leads to the contradiction $r=-2$.

Remarks. (1) For the case $\mathfrak{h}=\mathfrak{g}(n-1)$ we obtain a symmetric pair $(\mathfrak{g}, \mathfrak{h})$ which is irreducible and Einstein. This follows from $X Y \equiv 0$ and therefore $-2 \operatorname{Ric}(X, Y)=2 \operatorname{tr} \sigma(X, Y)=\operatorname{Kill}(X, Y)$

For the case $\mathfrak{h}=\mathfrak{g} 0(n-2)$ we obtain an irreducible reductive pair $(\mathfrak{g}, \mathfrak{h})$ but in this case $K=c$ is abelian. Thus in the notation of $[6$, p. 122] we have $c$ is spanned by the element $u \in \mathfrak{m}$. Using the explicit computations in [6], one can easily see that an $S$ exists which yields an irreducible Einstein connection.

(2) We shall now give a general test which shows that the above connection cannot be a connection of the first kind. Thus let $(\mathfrak{g}, \mathfrak{h})$ be a reductive pair with $\mathfrak{m}=\mathfrak{h} \perp$ relative to the Killing form where Kill $\mid \mathfrak{m} \times \mathfrak{m}$ is nondegenerate. The Ricci curvature for a connection of the first kind (i.e. $S=\lambda I)$ is given by

$$
\begin{aligned}
-4 \operatorname{Ric}_{1}(X, Y) & =2 \operatorname{Kill}(X, Y)-\operatorname{tr} L(X) L(Y) \\
& =\operatorname{tr} L(X) L(Y)+4 \operatorname{tr} \sigma(X, Y) .
\end{aligned}
$$

for $X, Y \in \mathfrak{m}$. Thus $-4 \operatorname{Ric}_{1}(X, Y)=\eta \operatorname{Kill}(X, Y)$ yields

$$
(1-\eta) \operatorname{tr} L(X) L(Y)=2(\eta-2) \operatorname{tr} \sigma(X, Y) .
$$


Now in the above example we have for all $P, Q \in K$ that $\operatorname{tr} \sigma(P, Q)=0$ while $\operatorname{tr} L(P) L(Q) \neq 0$ for some $P, Q \in K$; e.g. $P=Q \neq 0$. Thus from (4. 6) we must have $\eta=1$. But this gives $\operatorname{tr} \sigma(X, Y)=0$ for all $X, Y \in \mathfrak{m}$ which is impossible. This process generalizes when one considers linear combinations of $\operatorname{tr} L(X) L(Y)$ and $\operatorname{tr} \sigma(X, Y)$ for an admissible pseudo-Riemannian metric i.e. by demanding

$$
\lambda \operatorname{tr} L(X) L(Y)+\mu \operatorname{tr} \sigma(X, Y)=\eta \operatorname{Kill}(X, Y)
$$

(3) In the preceding example if we let $\mathfrak{h}_{1}=\mathfrak{h}+K \subset \mathfrak{g}$, then $\left(\mathfrak{g}, \mathfrak{h}_{1}\right)$ is a symmetric pair. Thus $\left[K_{2} K_{2}\right] \subset \mathfrak{h}_{1}$ so that $X_{44}=0$ so that one of the "trace forms" is zero. However more important for computing the trace forms is that $\left(\mathfrak{g}, \mathfrak{h}_{1}\right)$ is an irreducible pair i.e. ad $\mathfrak{h}_{1}$ acts irreducibly on $K_{2}$. Thus in an attempt to classify the reductive pairs $(\mathfrak{g}, \mathfrak{h})$ one could start with those pairs for which $\left(\mathfrak{g}, \mathfrak{h}_{1}\right)$ is an irreducible symmetric pair.

(4) More complicated computations show that the pair $(\mathfrak{g}, \mathfrak{h})$ with $\mathfrak{g}=\mathfrak{g} \mathfrak{D}(n)$ and $\mathfrak{h}=\mathfrak{g} \mathfrak{D}(p) \oplus \mathfrak{g} \mathfrak{D}(q)$ yields an irreducible Einstein space $(3 \leq p<$ $p+q \leq n-2)$. We consider $g$ as the set of skew-symmetric matrices

$$
\left(\begin{array}{lll}
F_{11} & F_{12} & F_{13} \\
-F_{12}{ }^{t} & h_{22} & F_{23} \\
-F_{13}{ }^{t} & -F_{23}{ }^{t} & h_{33}
\end{array}\right)
$$

and $\mathfrak{h}$ as the set of matrices

$$
\left(\begin{array}{lll}
0 & 0 & 0 \\
0 & h_{22} & 0 \\
0 & 0 & h_{33}
\end{array}\right)
$$

where $h_{22}$ is $p \times p$ and $h_{33}$ is $q \times q$. In $\mathfrak{m}=\mathfrak{h} \perp$ we note that

$$
\begin{aligned}
& K \text { is the set } \quad\left(\begin{array}{ccc}
F_{11} & 0 & 0 \\
0 & 0 & 0 \\
0 & 0 & 0
\end{array}\right) \\
& K_{1} \text { is the set } \quad\left(\begin{array}{ccc}
0 & 0 & 0 \\
0 & 0 & F_{23} \\
0 & -F_{23}{ }^{t} & 0
\end{array}\right)
\end{aligned}
$$




$$
\text { and } K_{2} \text { is the set }\left(\begin{array}{lll}
0 & F_{12} & F_{13} \\
-F_{12}{ }^{t} & 0 & 0 \\
-F_{13}{ }^{t} & 0 & 0
\end{array}\right)
$$

But note in this case $\left(\mathfrak{g}, \mathfrak{h}_{1}\right)$ is not irreducible or even a symmetric pair. However, if we let $p+q+r=n$ and let $\mathfrak{h}_{2}=\mathfrak{h}+K_{1}+K=\mathfrak{g} \mathfrak{g}(p+q)+\mathfrak{g} \mathfrak{o}(r)$, then $\left(\mathfrak{g}, \mathfrak{h}_{2}\right)$ is irreducible symmetric which simplifies computations and yields another possibility for a general classification process of reductive pairs $(\mathfrak{g}, \mathfrak{h})$.

Finally we give an example where the restricition of the Killing form is only nondegenerate and the algebra $K$ is not simple-actually $K=c$ is abelian. Thus let $g$ be the $n+1 \times n+1$ matrices of trace zero i.e. type $A_{n}$ and let $\mathfrak{h}$ be the $n \times n$ matrices of trace zero imbedded as follows [6, p. 121]. Let $e_{i j}$ denote the usual matrix basis where $i, j=1, \cdots, n+1$ and let $h_{k}=e_{k k}-e_{n+1 n+1}, k=1, \cdots, n$ which is a basis of a Cartan subalgebra. Let $\mathfrak{h}$ have basis

$$
\begin{aligned}
& e_{i j} \text { for } i, j=2, \cdots, n+1 \text { and } \\
& h_{k} \text { for } k=2, \cdots, n .
\end{aligned}
$$

Then noting that since $\operatorname{Kill}_{\mathfrak{g}}(X, Y)=2(n+1) \operatorname{tr} X Y$ for all $X, Y \in \mathrm{g}$ we see that Kill $\mid \mathfrak{h} \times \mathfrak{h}$ is nondegenerate so we let $\mathfrak{m}=\mathfrak{h} \perp$. Thus $\mathfrak{m}$ has a basis

$$
\begin{aligned}
& e_{1 j}, e_{j 1} \text { for } j=2, \cdots, n+1 \text { and } \\
& u=-n h_{1}+h_{2}+\cdots+h_{n}
\end{aligned}
$$

and we have the multiplication relations [6]

$$
\begin{aligned}
& u e_{1 k}=-(n+1) e_{1 k}, u e_{k 1}=(n+1) e_{k 1} \\
& e_{1 r} e_{1 q}=e_{q 1} e_{p 1}=0, e_{1 p} e_{q 1}=\frac{1}{n} \delta_{p q} u .
\end{aligned}
$$

We note that $K=\mathrm{Ru}$ i.e. $K^{\prime}=0, K_{1}=0$ and $K_{2}=L_{1} \dot{+} L_{2}$ where $L_{1}$ (resp. $L_{2}$ ) is spanned by the $e_{1 j}$ (resp. $e_{j 1}$ ) and the $L_{i}$ are ad $\mathfrak{h}_{1}$-irreducible where $\mathfrak{h}_{1}=\mathfrak{h}+K=\mathfrak{h}_{2}$. Thus $\left(\mathfrak{g}, \mathfrak{h}_{2}\right)$ is a reducible symmetric space but we can still compute the various trace forms.

Next we compute the equations (3.9)-(3. 12). Thus since $K^{\prime}=K_{1}=0$, we have

$$
\eta=s_{1} s_{4}^{-2}
$$


which uses $\operatorname{tr} L(u) L(u) \neq 0$, (see (4. 9)).

Next note for $X \in K_{2}$ that in (3. 8) we have $X_{24}=X_{34}=0=X_{42}=X_{43}=X_{44}$. Thus from (3. 12) we have

$$
\begin{aligned}
& 2\left(\eta s_{4}^{-2}\right) \operatorname{tr} \sigma(X, Y)+\left(\eta s_{4}^{-2}+s_{1}^{-1} s_{4}\right) \operatorname{tr} X_{41} Y_{14} \\
& \quad+\left(\eta s_{4}^{-2}+2 s_{1} s_{4}^{-1}-s_{4} s_{1}^{-1}\right) \operatorname{tr} X_{14} Y_{41} \\
& =0
\end{aligned}
$$

But a straight forward computation shows that for $Z=z_{0} u+\sum z_{1 k} e_{1 k}+\sum z_{k 1}^{\prime} e_{k 1} \in \mathfrak{m}$,

$$
L(Z)=\left(\begin{array}{lll}
0 & (n+1) z & -(n+1) z^{\prime} \\
-\frac{1}{n}{ }^{t} z^{\prime} & -(n+1) z_{0} I & 0 \\
\frac{1}{n}{ }^{t} z & 0 & (n+1) z_{0} I
\end{array}\right)
$$

where $z=\left(z_{12} \cdots z_{1 n+1}\right), z^{\prime}=\left(z_{21}^{\prime} \cdots z_{n+11}^{\prime}\right)$ and ${ }^{t} z$ denotes transpose. Thus for $U, V \in \mathfrak{m}$ we obtain

$$
\begin{aligned}
\operatorname{tr} L(U) L(V)=- & \frac{2(n+1)}{n}\left[u\left({ }^{t} v^{\prime}\right)+v\left({ }^{t} u^{\prime}\right)\right] \\
& +2 n(n+1)^{2} u_{0} v_{0} \\
=- & \frac{2(n+1)}{n} \sum\left(u_{i} v_{i}{ }^{\prime}+v_{i} u_{i}{ }^{\prime}\right) \\
& +2 n(n+1)^{2} u_{0} v_{0} .
\end{aligned}
$$

In particular for $X, Y \in K_{2}$ we have since $X_{14}=(n+1)\left(x,-x^{\prime}\right)$ and $X_{41}=$ $\frac{1}{n}^{t}\left(-x^{\prime}, x\right)$ that

$$
\begin{aligned}
\operatorname{tr} X_{14} Y_{41} & =\operatorname{tr} X_{41} Y_{14} \\
& =\frac{1}{2} \operatorname{tr} L(X) L(Y) .
\end{aligned}
$$

Next using $\operatorname{tr} L(X) L(Y)+2 \operatorname{tr} \sigma(X, Y)=\operatorname{Kill}(X, Y)=2(n+1) \operatorname{tr} X Y=-2 n \operatorname{tr} X_{14} Y_{41}$ $=-n \operatorname{tr} L(X) L(Y)$ we obtain

$$
\operatorname{tr} \sigma(X, Y)=-\frac{1}{2}(n+1) \operatorname{tr} L(X) L(Y) .
$$

Thus equation (4. 8) becomes 


$$
\begin{aligned}
0=[ & -(n+1)\left(\eta s_{4}-2\right)+\frac{1}{2}\left(\eta s_{4}-2+s_{1}^{-1} s_{4}\right) \\
& \left.+\frac{1}{2}\left(\eta s_{4}-2+2 s_{1} s_{4}^{-1}-s_{4} s_{1}^{-1}\right)\right] \operatorname{tr} L(X) L(Y)
\end{aligned}
$$

which yields

$$
n \eta s_{4}{ }^{2}-2 n s_{4}-s_{1}=0 .
$$

Using this and (4.7) we obtain the solution

$$
s_{4}=\frac{n-1}{2 n} s_{1} \neq 0
$$

From this $2 s_{4}=s_{1}$ is impossible so we obtain irreducibility.

Remarks. (1) Using the previous remark and (4.6) we see the above connection cannot be of the first kind since $\operatorname{tr} \sigma(u, u)=0$ while $\operatorname{tr} L(u) L(u) \neq 0$.

(2) If $\mathfrak{h}$ is of type $A_{r}$ with $r \leq n-2$ and imbedded in $g$ similar to the above example, then we also obtain an irreducible Einstein connection. In this case $K=c \oplus K^{\prime}$ where $c=R u$ is one dimensional and $K^{\prime}$ is simple of type $A_{n-r-1}$.

These and other examples led to the conjecture in the Introduction. It appears that the solution to (3.3) will have to be done by other methods than used above but that irreduciblity can be done algebraically.

\section{REFERENCES}

[ 1 ] D. Husemoller, Fiber Bundles. McGraw-Hill Company, 1967.

[2] N. Jacobson, Lie Algebras, John Wiley, 1962.

[ 3 ] B. Kostant, On holonomy and homogeneous spaces, Nagoya Math. Jol. vol. 12 (1957), 31-54.

[4] O. Loos, Symmetric Spaces, W.A. Benjamin, to appear.

[5] K. Nomizu, Invariant affine connections on homogeneous spaces, Amer. Math. Jol., vol. 76 (1954), 33-65.

[6] A. Sagle, A note on simple anti-commutative algebras obtained from reductive homogeneous spaces, Nagoya Math. Jol., vol. 31 (1968), 105-124.

[ 7 ] A Sagle, On anti-commutative algebras and homogeneous spaces, Jol. Math. and Mech., vol. 16 (1967), 1381-1393.

[8] A. Sagle, On homogeneous spaces, holonomy and nonassociative algebras, Nagoya Math. Jol., vol. 32 (1968), 373-394. 
[ 9 ] A. Sagle and D. J. Winter, On homogeneous spaces and reductive subalgebras of simple Lie algebras, Trans. Amer. Math. Soc., vol. 128 (1967), 142-147.

[10] E. Spanier, Algebraic Topology, McGraw-Hill Company, 1966.

[11] J. Wolf, The geometry and structure of isotropy-irreducible homogeneous spaces, Acta Mathematica, vol. 120 (1968), 59-148.

University of Minnesota 